\title{
Elevated circulating adenosine deaminase activity in women with preeclampsia: association with pro-inflammatory cytokine production and uric acid levels
}

\author{
Vanessa S. Giorgi ${ }^{a}$, Steven S. Witkin ${ }^{\mathrm{b}}$, Camila F. Bannwart-Castro ${ }^{\mathrm{c}}$, Maria Salete Sartori ${ }^{\mathrm{c}}$, \\ Mariana Romão-Veiga ${ }^{a}$, Vera Therezinha M. Borges ${ }^{c}$, José Carlos Peraçoli ${ }^{c}$, Maria Terezinha Peraçoli ${ }^{\mathrm{a}, *}$ \\ a Department of Microbiology and Immunology, Institute of Biosciences, São Paulo State University, Botucatu, São Paulo, Brazil \\ ${ }^{\mathrm{b}}$ Department of Obstetrics and Gynecology, Weill Cornell Medicine, New York, New York, USA \\ ${ }^{\mathrm{c}}$ Department of Gynecology and Obstetrics, São Paulo State University, Botucatu, São Paulo, Brazil
}

\section{A R T I C L E I N F O}

\section{Article history:}

Received 21 July 2016

Accepted 20 September 2016

Available online 21 September 2016

\section{Keywords:}

Adenosine deaminase

IL-1 $\beta$

NF-kB

Preeclampsia

TNF- $\alpha$

Uric acid

\begin{abstract}
A B S T R A C T
Objective: Preeclampsia is a specific disorder of human pregnancy that is associated with hyperuricemia and higher levels of pro-inflammatory cytokines. Adenosine deaminase (ADA) is an enzyme present in all human tissues, and is considered an indicator of cellular inflammation. In the present study we assess whether adenosine deaminase (ADA) activity is altered in women with preeclampsia (PE) and contributes to elevated levels of uric acid and pro-inflammatory cytokine production.

Study design: The population studied consisted of 60 women with PE, 30 normotensive pregnant women (NT) and 20 non-pregnant women (NP). Uric acid concentration and ADA activity were determined in the serum. Peripheral blood mononuclear cells (PBMCs) were isolated and evaluated for intracellular nuclear transcription factor kappa B (NF- $\kappa B$ ) levels and for endogenous tumor necrosis factor- $\alpha$ (TNF- $\alpha$ ) and interleukin-1 $\beta$ (IL-1 $\beta$ ) production. The data were evaluated with parametric or non-parametric tests with significance set at $P<0.05$.

Results: ADA levels were higher in the PE group compared with the NT and NP groups $(P<0.001)$. A positive correlation between ADA and uric acid levels was identified in women with $\mathrm{PE}(P<0.001)$. Endogenous production of IL-1 $\beta$ and TNF- $\alpha$, as well as intracellular NF- $\kappa B$ levels, were higher in PBMCs from the PE group than from NT and NP women $(P<0.01)$ and correlated with the ADA concentration in preeclamptic women $(P<0.01)$.

Conclusion: An elevation in ADA activity in women with PE may contribute to their increased levels of uric acid and pro-inflammatory immune activity.

(c) 2016 International Society for the Study of Hypertension in Pregnancy. Published by Elsevier B.V. All rights reserved.
\end{abstract}

\section{Introduction}

Preeclampsia (PE) is a dangerous complication of pregnancy that is responsible for a high proportion of maternal and infant morbidity and mortality [1]. The disease affects $2-10 \%$ of pregnant women [2], and was recently redefined as hypertension present after 20 weeks of gestation combined with proteinuria ( $>300 \mathrm{mg} /-$ day) and other maternal organ dysfunction, such as renal insufficiency, liver involvement, neurological or haematological

\footnotetext{
* Corresponding author at: Department of Microbiology and Immunology, Institute of Biosciences of Botucatu, Rubião Junior s/n, UNESP, Botucatu, São Paulo CEP 18618-970, Brazil.

E-mail address: peracoli@ibb.unesp.br (M.T. Peraçoli).
}

complications, uteroplacental dysfunction, or fetal growth restriction $[3,4]$.

Although the etiology of PE remains unclear, the associated pathogenesis has been extensively studied. Excessive activation of leukocytes in PE is associated with exaggerated innate and adaptive immune responses that may interfere with normal pregnancy progression [5]. Increased production of pro-inflammatory cytokines and activation of maternal endothelial cells result in a systemic and diffuse endothelial cell dysfunction that is the fundamental pathophysiological feature of PE [1]. Another important contributor to development of this disorder is oxidative stress; the upregulated production of reactive oxygen species (ROS), superoxide anion and hydrogen peroxide induces enhanced lipid peroxidation [6]. A higher level of activation of nuclear transcription factor- $\kappa B$ 
(NF- $\mathrm{KB})$, responsible for the transcription of genes related to inflammation, is also evident in mononuclear lymphoid cells of preeclamptic women and results in enhanced release of the primary pro-inflammatory cytokines, tumor necrosis factor- $\alpha$ (TNF$\alpha)$ and interleukin- $1 \beta$ (IL-1 $\beta$ ) $[7,8]$. Several studies have suggested that a high concentration of circulating TNF- $\alpha$ is associated with more severe clinical manifestations of PE $[9,10]$.

To down-regulate deleterious increases in immune responses, adenosine nucleotides are released at inflammatory and ischemic sites into the extracellular milieu where they are converted to adenosine by ectonucleases. Concomitantly, intracellular ATP is also dephosphorylated and adenosine is released from the cells. The accumulated adenosine binds to specific receptors on macrophages, neutrophils and $\mathrm{T}$ lymphocytes triggering an increase in intracellular levels of cyclic AMP and results in the inhibition of pro-inflammatory cytokine production $[11,12]$. Adenosine deaminase (ADA) is the enzyme that decreases extracellular adenosine levels by catalyzing its hydrolytic deamination to inosine [13]. Addition of ADA to plasma from newborn infants has been shown to result in an enhanced capacity of mononuclear cells to produce TNF- $\alpha$ [14].

Serum ADA activity is reduced in pregnant as compared to nonpregnant women [15] suggesting an increased need for adenosinemediated down-regulation of pro-inflammatory immunity during gestation. The association between elevated ADA activity and PE was first reported by Yoneyama et al. [16-18]. Elevated levels of ADA in maternal plasma, umbilical cord blood and the placenta were associated with development of PE, but not with the severity of the disease [19]. A correlation between elevated ADA and myeloperoxidase activities with disease severity and neonatal outcomes in preeclamptic women has also been reported [20].

In pregnant women with PE an elevated serum level of uric acid has been associated with increased production of proinflammatory cytokines by mononuclear cells in peripheral blood [21]. Superoxide anion, the inducible $70 \mathrm{kDa}$ heat shock protein and TNF- $\alpha$ production by monocytes have also been associated with uric acid levels in PE [7,9]. Thus, high circulating uric acid levels may directly contribute to the pathogenesis of PE by its promotion of inflammation [22].

Uric acid is the final breakdown product from metabolism of the purines ATP, ADP, AMP, adenosine, inosine and hypoxanthine. The origins of high purine levels, increases in purine metabolism as well as the mechanism leading to elevated levels of uric acid in preeclamptic women are unknown. It is possible that trophoblast microparticles released into the circulation as a consequence of placental damage in preeclamptic women may provide the major substrate for purine metabolism and uric acid formation $[23,24]$.

The present study evaluated whether ADA activity in sera of preeclamptic women was related to enhanced intracellular NF$\kappa \mathrm{B}$ activity in peripheral blood mononuclear cells (PBMCs), endogenous TNF- $\alpha$ and IL- $1 \beta$ production by PBMCs and serum levels of uric acid. Elucidation of these associations would strongly suggest the major involvement of adenosine metabolism in development of PE-associated pathology.

\section{Materials and methods}

\subsection{Patients and controls}

The study group consisted of 60 primiparous women without a previous history of hypertension or obstetric and medical complications, admitted to the Obstetric Unit of Botucatu Medical School, Botucatu, SP, Brazil, with a diagnosis of PE. Preeclampsia was defined as a persistent elevated blood pressure value $(\geqslant 140 / 90 \mathrm{mmHg}$ ) evaluated on two occasions $2 \mathrm{~h}$ apart after
20 weeks of gestation and proteinuria ( $\geqslant 300 \mathrm{mg} / 24 \mathrm{~h}$ in urine collected during $24 \mathrm{~h}$ ) in women with no previous history of hypertension [2]. Thirty normotensive (NT) primiparous women with an uncomplicated pregnancy who were non-proteinuric were recruited and matched for gestational age at time of sampling with the preeclamptic group. These women remained normotensive and non-proteinuric until the end of gestation. Gestational age was calculated from the last menstrual period and confirmed by early (<12 weeks gestation) ultrasound examination. Twenty nonpregnant (NP) women were volunteer donors of the Blood Bank from the Hemocenter of the Botucatu Medical School, Botucatu, SP, Brazil and were included as additional controls. Proteinuria in 24-h urine was measured by a colorimetric method, the Technicon RAXT automation system, and uric acid in serum was assessed by uric acid enzymatic Trinder (Biotrol Diagnostic, Bridgewater, NJ, USA) in the Clinical Laboratory of Botucatu Medical School, Botucatu, SP, Brazil. Exclusion criteria included multiple gestation, illicit drug use, and preexisting medical conditions such as diabetes, chronic hypertension, and renal disease. For NP women the same exclusion criteria of pregnant women was utilized, except for gestation characteristics. The study was approved by the Ethics Committee of the Botucatu Medical School, and the written informed consent was obtained from all women involved in the study. (Protocol number $3443 / 10$ ). For pregnant women with age below 18 years old the written informed consent was obtained from their parents or guardians.

\subsection{Determination of adenosine deaminase serum activity}

Ten milliliters of blood was collected by venipuncture from the antecubital vein of PE, NT and NP women, and after clot formation the samples were centrifuged at $4{ }^{\circ} \mathrm{C}$ for $10 \mathrm{~min}$ at $1200 \mathrm{~g}$. The serum fraction was stored in aliquots at $-80^{\circ} \mathrm{C}$ until the analyses were performed. For ADA activity evaluation preeclamptic patients were also classified as early-onset PE $(n=30)$ or late-onset PE $(\mathrm{n}=30)$ according to whether disease manifestations occurred before or from the 34th week of gestation, respectively. ADA activity was determined by automation (Vitros System Model 5.1/FS) and a commercial kit (Diagnostica Genbiotech, Brazil) following the manufacturer's instructions. The kit, a non-radioactive colorimetric method, is based on the enzymatic deamination of adenosine to inosine to hypoxanthine by the enzyme purine nucleoside phosphorylase. Hypoxanthine is converted into uric acid and hydrogen peroxide $\left(\mathrm{H}_{2} \mathrm{O}_{2}\right)$ by xanthine oxidase. $\mathrm{H}_{2} \mathrm{O}_{2}$ reacts with N-ethyl-N-(2-hydroxy-3-sulfopropyl)-3-methylaniline (EHSPT) and 4-amino antipyrine (4-AA) in the presence of peroxidase and generates the quinone dye, which is measured by a colorimetric method. One ADA unit is defined as the amount required to generate $1 \mathrm{umol}$ of inosine per minute at $37^{\circ} \mathrm{C}$. Results were expressed in IU/L.

\subsection{Peripheral blood mononuclear cell (PBMC) culture}

PBMCs were isolated from heparinized venous blood by density gradient centrifugation on Histopaque, as described previously [7]. PBMC at a concentration of $5 \times 10^{6}$ cells/mL in RPMI 1640 tissue culture medium (Gibco Laboratories, Grand Island, NY, USA) containing $2 \mathrm{mM}$ L-glutamine, 10\% heat-inactivated fetal calf serum, $20 \mathrm{mM}$ HEPES, and $40 \mu \mathrm{g} / \mathrm{mL}$ gentamicin were dispensed $(100 \mu \mathrm{L}$ per well) into 24-well sterile flat bottom plates (Nalge Nunc Int, Rochester, NY, USA). The cells were cultured without exogenous stimulation at $37{ }^{\circ} \mathrm{C}$ and $5 \% \mathrm{CO} 2$ for 30 min to assay for intracellular NF- $\kappa$ B activity and for $18 \mathrm{~h}$ to assay for cytokine release into the culture medium. 


\subsection{Determination of TNF- $\alpha$ and $I L-1 \beta$}

TNF- $\alpha$ and IL- $1 \beta$ levels were measured in supernatants of the PBMC cultures by enzyme-linked immunosorbent assay (ELISA), using Quantikine ELISA kits (R\&D Systems, Minneapolis, MN, USA), according to the manufacturer's instructions. The sensitivity limit of the assay for TNF- $\alpha$ and IL- $1 \beta$ was $10 \mathrm{pg} / \mathrm{mL}$.

\subsection{Detection of $N F-\kappa B$}

Nuclear transcription factor kappa $\mathrm{B}(\mathrm{NF}-\kappa \mathrm{B})$ was determined in PBMC lysates. Cells were washed with ice-cold saline buffer containing $10 \%$ endotoxin-free fetal calf serum and then lysed using a nuclear extract kit (Cayman Chemical Co., Ann Arbor, MI, USA) according to the manufacturer's instructions. The level of p65NF$\kappa \mathrm{B}$ in nuclear extracts was determined using a transcription factor ELISA kit (Cayman Chemical). Protein concentrations in nuclear extracts were determined by Lowry's method [25], and p65NF$\kappa \mathrm{B}$ was expressed as $\mu \mathrm{g} / \mu \mathrm{g}$ total nuclear protein.

\subsection{Statistical analysis}

The data were evaluated with parametric or non-parametric tests, using Prisma Statistical software 9 version 5.0 (GraphPad San Diego, Calif., U.S.A.). Correlation analyses were performed by Spearman rank correlations. A p value $<0.05$ was considered significant.

\section{Results}

\subsection{Characteristics of pregnant and non-pregnant women}

Table 1 lists characteristics of the three groups studied. No statistical differences were evident between groups with respect to maternal or gestational age. Proteinuria was higher in the PE group than in the NT group $(P<0.05)$. Systolic and diastolic blood pressure and the uric acid serum concentration were higher in women with PE as compared to the NT and NP women $(P<0.05)$.

\section{Table 1}

Clinical characteristics of pregnant women with preeclampsia, normotensive pregnant women and non-pregnant women.

\begin{tabular}{|c|c|c|c|}
\hline Characteristics & $\begin{array}{l}\text { Pregnant women } \\
\text { with preeclampsia } \\
(\mathrm{n}=60)\end{array}$ & $\begin{array}{l}\text { Normotensive } \\
\text { pregnant women } \\
(\mathrm{n}=30)\end{array}$ & $\begin{array}{l}\text { Non-pregnant } \\
\text { women } \\
(\mathrm{n}=20)\end{array}$ \\
\hline Age (years) & $\begin{array}{l}26 \\
(13-41)\end{array}$ & $\begin{array}{l}23 \\
(15-39)\end{array}$ & $\begin{array}{l}25 \\
(19-39)\end{array}$ \\
\hline $\begin{array}{l}\text { Gestational age } \\
\text { (weeks) }\end{array}$ & $\begin{array}{l}35 \\
(24-40)\end{array}$ & $\begin{array}{l}34 \\
(24-34)\end{array}$ & $\mathrm{N} / \mathrm{A}$ \\
\hline Systolic Blood & $160^{\mathrm{a}}$ & 110 & 114 \\
\hline $\begin{array}{l}\text { Pressure } \\
(\mathrm{mmHg})\end{array}$ & $(140-200)$ & $(90-112)$ & $(100-120)$ \\
\hline $\begin{array}{l}\text { Diastolic Blood } \\
\text { Pressure } \\
\text { (mmHg) }\end{array}$ & $\begin{array}{l}110^{\mathrm{a}} \\
(90-120)\end{array}$ & $\begin{array}{l}69 \\
(63-70)\end{array}$ & $\begin{array}{l}70 \\
(65-80)\end{array}$ \\
\hline $\begin{array}{l}\text { Proteinuria } \\
\qquad(\mathrm{mg} / 24 \mathrm{~h})\end{array}$ & $\begin{array}{l}1205^{\mathrm{b}} \\
(300-22,520)\end{array}$ & $<300$ & $\mathrm{~N} / \mathrm{A}$ \\
\hline $\begin{array}{l}\text { Uric acid (mg/ } \\
\quad \text { dL) }\end{array}$ & $\begin{array}{l}6.1^{\mathrm{a}} \\
(4.5-10.1)\end{array}$ & $\begin{array}{l}3.8 \\
(2.2-4.6)\end{array}$ & $\begin{array}{l}4.1 \\
(2.8-4.8)\end{array}$ \\
\hline
\end{tabular}

Data are presented as median, with the minimum and maximum values in parentheses.

$\mathrm{N} / \mathrm{A}=$ not applicable.

a $(P<0.05) v s$ normotensive pregnant and non-pregnant groups (Kruskal-Wallis test)

b $(P<0.05)$ vs normotensive pregnant group (Mann-Whitney $U$ test)

\subsection{ADA activity}

ADA activity was increased in the serum of women with PE compared to those in the NT and NP groups $(P<0.001)$. No significant differences were detected between the NT and NP women (Fig. 1A), and between early-onset PE and late-onset PE (Fig. 1B). A positive correlation between ADA activity and uric acid levels in preeclamptic women was observed (Spearman $r=0.6425$; $P<0.0001$ ) (Fig. 2A). There was no correlation between ADA and uric acid levels in the NT $(r=0.2106 ; P=0.2639)$ (Fig. 2B), or NP women ( $r=0.2308 ; P=0.3276$ ) (Fig. 2 C).

\subsection{TNF- $\alpha$, IL-1 $\beta$ levels and NF- $\kappa B$ activity}

Endogenous production of the pro-inflammatory cytokine TNF$\alpha$ by PBMC was significantly higher $(P<0.01)$ in PBMCs from women with PE $(98.6 \mathrm{pg} / \mathrm{mL} \pm 12.1)$ in comparison to NT (34.5 pg/mL \pm 6.1 ) and NP women (29.7 pg/mL \pm 7.6) (Fig. 3A). IL$1 \beta$ production was also significantly higher $(P<0.01)$ in the PE group (1598.2 $\mathrm{pg} / \mathrm{mL} \pm 120.8$ ), as compared with the NT group (1123.8 pg/mL \pm 157.6 ) and NP group (1049.5 pg/mL \pm 123.8 ) (Fig. 3B). The activity of endogenous NF- $\kappa B$, represented by p65NF- $\kappa$ B detected in nuclear extract of PBMCs, was significantly higher $(P<0.01)$ in pregnant women with $\mathrm{PE}(0.63 \mu \mathrm{g} / \mu \mathrm{g}$ protein \pm 0.04$)$ than in NT $(0.24 \mu \mathrm{g} / \mu \mathrm{g}$ protein \pm 0.02$)$ and $\mathrm{NP}$ $(0.22 \mu \mathrm{g} / \mu \mathrm{g}$ protein \pm 0.03$)$ women (Fig. $3 C$ ). Correlation analyses between ADA and NF- $\kappa B$, IL- $1 \beta$ and TNF- $\alpha$ for the three study groups are shown in Table 2. Positive correlations between ADA activity and NF- $\kappa B$, IL- $1 \beta$ and TNF- $\alpha$ concentration were detected in preeclamptic women $(P<0.01)$. Correlation between ADA and NK- $\kappa \mathrm{B}$ activity was also observed in the NP group $(P<0.05)$. No correlations between these parameters were observed in the NT group.

\section{Discussion}

ADA activity was elevated in sera from women with preeclampsia as opposed to normotensive pregnant women and nonpregnant women. Furthermore, ADA and hyperuricemia were highly correlated in PE. This strongly suggests that the degradation of circulating adenosine as well as other purine compounds occurred to a greater extent in women with PE than in other women and may contribute to development of hyperuricemia. ADA activity was also positively correlated with elevated NF- $\kappa$ B levels and pro-inflammatory cytokine production by PBMCs in women with PE. This is likely a consequence of increased degradation of anti-inflammatory adenosine. Thus, the degradation of adenosine by ADA and the subsequent accumulation of uric acid appear likely to be a contributor to the elevated systemic inflammatory response characteristic of PE.

The severity of PE and subsequent maternal and perinatal outcome has been shown to correlate with hyperuricemia $[22,26]$. Uric acid is recognized as a host-derived danger-associated molecular pattern (DAMP), since it is released primarily under nonphysiological conditions. It binds to the Nod-like receptor (NLR), NLRP3 [27], and promotes formation of the inflammasome, the intracellular cytoplasmic structure that mediates the processing and release of biologically active IL-1 $\beta$ and IL-18 [28,29]. Recently, we demonstrated that monocytes from preeclamptic women have enhanced endogenous NLRP3 inflammasome activity and release high levels of IL-1 $\beta$ and IL-18. Moreover, monocyte stimulation by exogenous monosodium urate led to NLRP3 and caspase- 1 activation and induced IL-1 $\beta$ and IL-18 release from monocytes of women with PE [30]. The reduction in adenosine levels would fur- 

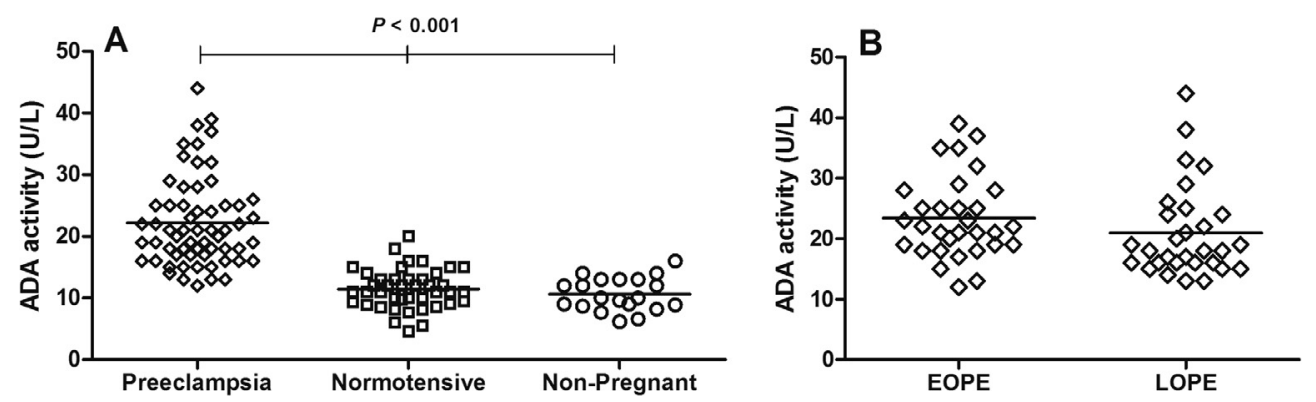

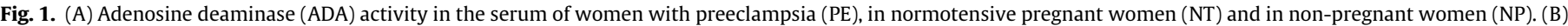

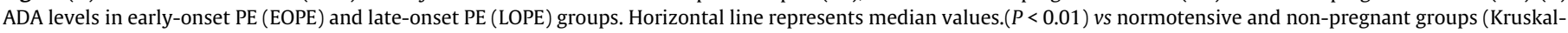
Wallis test).
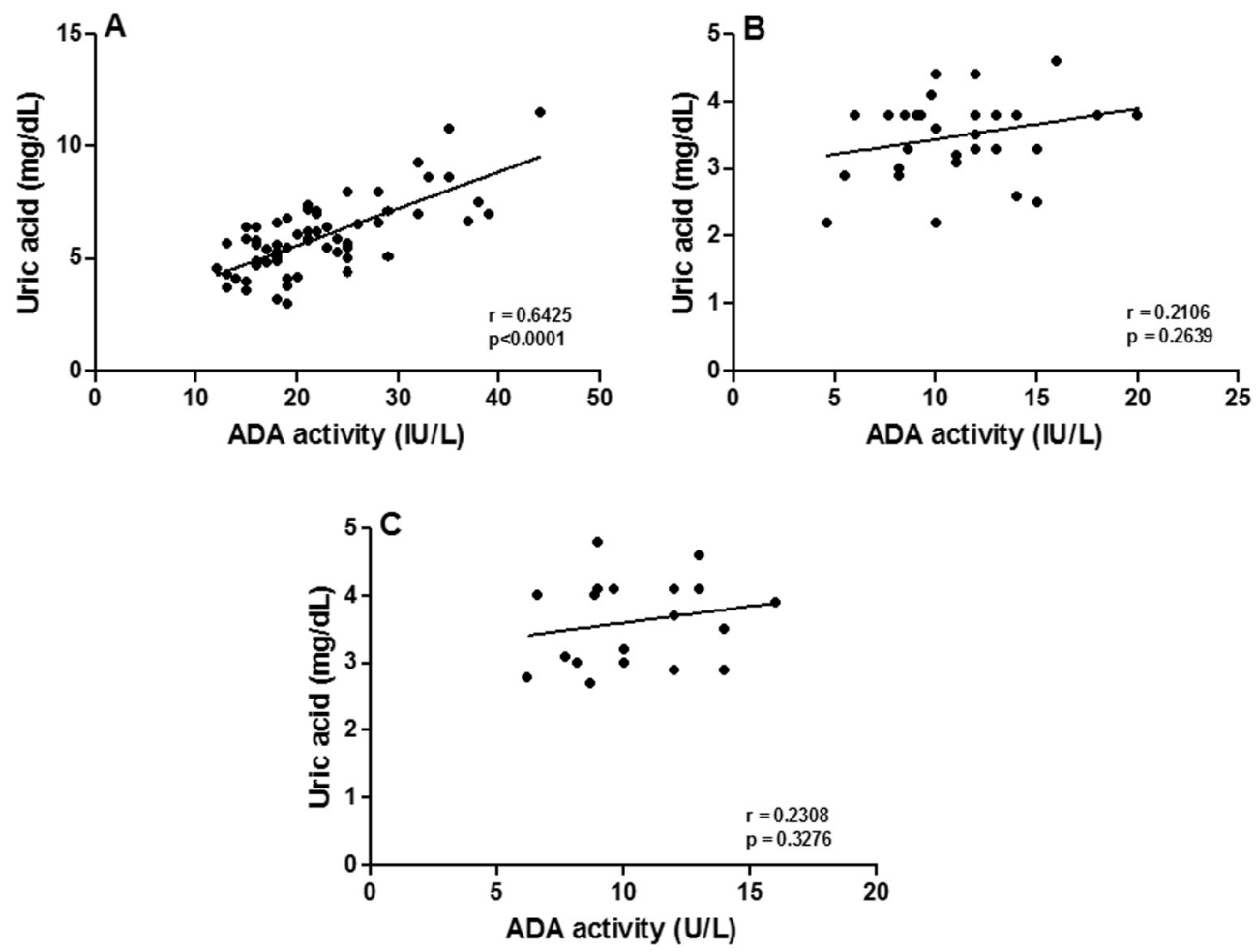

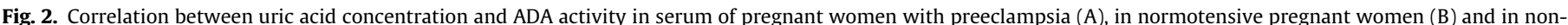
pregnant women (C).

ther potentiate uric acid-mediated pro-inflammatory immune system activation.

The extent and duration of inflammasome activity is regulated by autophagy [31]. Inflammasomes are engulfed by autophagosomes and delivered to lysosomes for digestion. Recently, the ability of sera from women with preeclampsia, but not sera from women with normotensive pregnancies, to induce autophagy in PBMCs was shown to diminish with advancing gestational age [32]. It is interesting to speculate whether the high ADA levels in sera from preeclamptic women contributed to this alteration in autophagy induction. NF- $\kappa B$, whose level is increased in association with increased ADA concentration, is a known inhibitor of autophagy [33]. A lowered capability to down-regulate inflammasome activity by a reduction in autophagy induction secondary to elevated ADA levels provides an additional mechanism leading to elevated pro-inflammatory immune system activation in PE.

The adenosine concentration in plasma from newborns is threefold higher than in plasma from adults [14] and adenosine has been shown, at least in a guinea pig model, to pass from the mater- nal to the fetal circulation [34]. Thus, the elevation in circulating ADA levels in women with PE may lead to a reduction in fetal adenosine levels and a subsequent increased magnitude in the fetal immune response to adverse stimuli. This may lead to alterations in developmental programs as well as increase the likelihood of a preterm delivery. In support of the influence of ADA on fetal immunity it was shown that when exogenous ADA was added to unfractionated ex vivo amniotic fluid cultures there was an enhanced production of TNF- $\alpha$ [35].

While the reason(s) for elevated ADA in the circulation of women with PE remain incompletely determined, it appears that individual variations in the level and/or activity of ADA may very well influence susceptibility to develop PE. Studies to determine the involved mechanism leading to ADA over-production may lead to development of novel protocols to prevent or effectively treat this debilitating problem of pregnancy.

A strength of the present study is that all PE subjects were from a dedicated clinic with a long history of PE diagnosis and treatment and experience in the measurement of immune parameters in this 

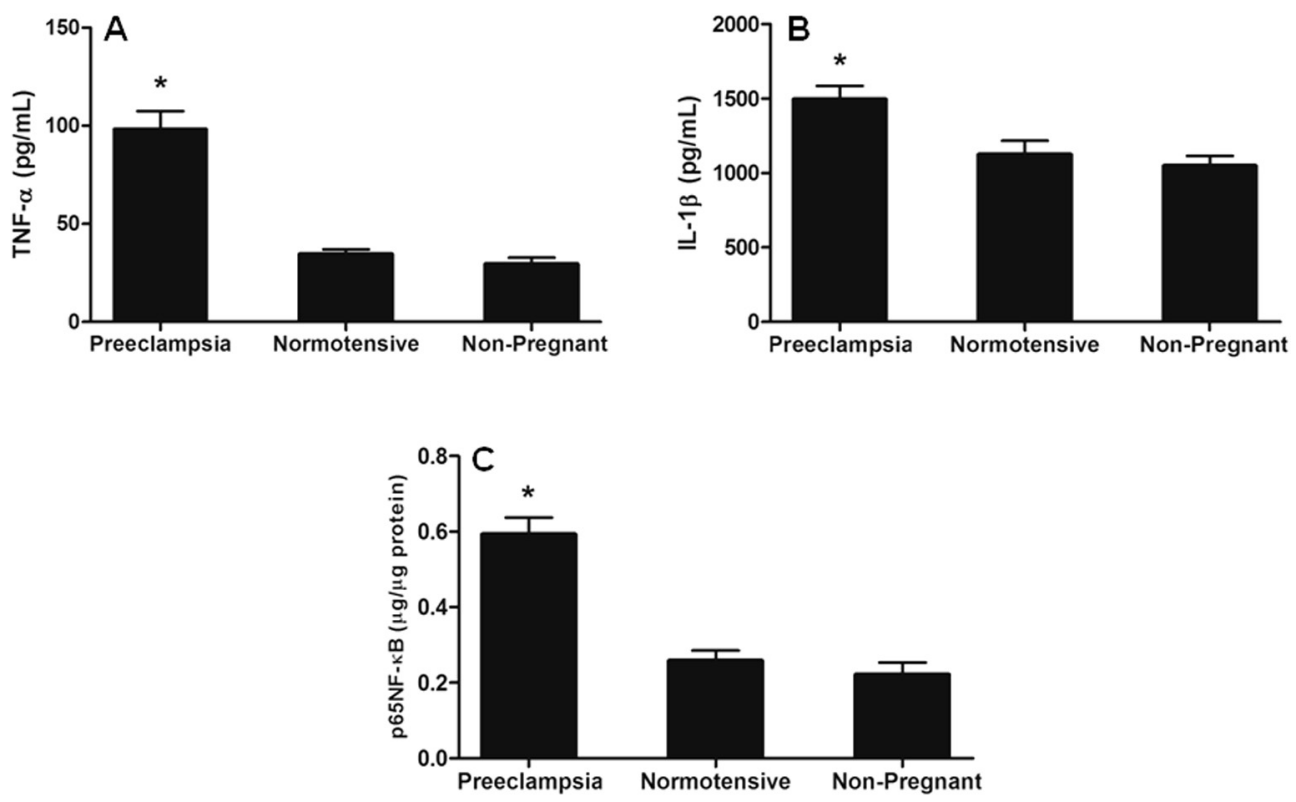

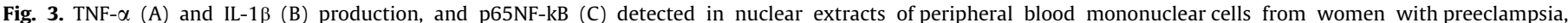
normotensive pregnant and non-pregnant women. Results are expressed as mean + SD. ${ }^{*}(P<0.01)$ vs normotensive and non-pregnant groups (ANOVA).

Table 2

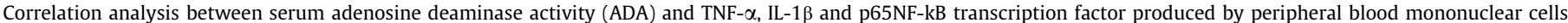
from preeclamptic, normotensive and nonpregnant women.

\begin{tabular}{|c|c|c|c|c|c|c|}
\hline Correlation ADA (IU/L) with & Preeclampsia $\mathrm{r}$ & $P$ & Normotensive $\mathrm{r}$ & $P$ & Non-pregnant $\mathrm{r}$ & $P$ \\
\hline TNF- $\alpha(p g / m L)$ & 0.5671 & 0.0011 & 0.3564 & 0.0951 & 0.4047 & 0.0787 \\
\hline $\mathrm{IL}-1 \beta(\mathrm{pg} / \mathrm{mL})$ & 0.6211 & 0.0035 & 0.3108 & 0.1704 & 0.3141 & 0.1775 \\
\hline NF-kB ( $\mu g / \mu g$ protein $)$ & 0.5774 & 0.0008 & 0.3946 & 0.0851 & 0.5412 & 0.0167 \\
\hline
\end{tabular}

population. The two control subject groups, normotensive pregnant women and non-pregnant women, were from the same city and matched for age and ethnicity, thus minimizing the influence of extraneous variables on the measurements. A limitation of the present study is the absence of direct measurement of adenosine concentrations in our study subjects. This protocol was not available at the time of our investigation and needs to be investigated in future studies. It also remains to be determined whether measurement of ADA or adenosine levels in women prior to their becoming pregnant or at early gestational stages will predict their likelihood of developing PE later in their pregnancy.

\section{Conclusion}

The association between an elevated serum concentration of $A D A$, increased production of pro-inflammatory cytokines and development of hyperuricemia suggests that ADA plays a central role in induction of the deleterious systemic inflammatory response in women with $\mathrm{PE}$.

\section{Conflicts of interest}

The authors state that they have no financial or commercial conflicts of interest.

\section{Contribution to authorship}

SSW and MTP conceived and design the study. VSG, CFBC, MSS and MRV performed the experiments. VTB and JCP selected preg- nant and non-pregnant women. MTP, JCP and SSW drafted the manuscript. All authors approved the manuscript for publication.

\section{References}

[1] R. Raghupathy, Cytokines as key players in the pathophysiology of preeclampsia, Med. Princ. Pract. 22 (suppl 1) (2013) 8-19.

[2] ACOG Practice Bulletin no. 33, Diagnosis and management of preeclampsia and eclampsia, Obstet. Gynecol. 99 (2002) 159-167.

[3] A.L. Tranquilli, G. Dekker, L. Magee, J. Roberts, B.M. Sibai, W. Steyn, et al., The classification, diagnosis and management of the hypertensive disorders of pregnancy: a revised statement from the ISSHP, Pregnancy Hypertens. 4 (2014) 97-104.

[4] B.W. Mol, C.T. Roberts, S. Thangaratinam, L.A. Magee, C.J. de Groot, G.J. Hofmeyr, Pre-eclampsia, Lancet S0140-6736 (2015) 70-77.

[5] C.A. Lok, J. Jebbink, R. Nieuwland, M.M. Faas, K. Boer, A. Sturk, et al., Leukocyte activation and circulating leukocyte-derived microparticles in preeclampsia, Am. J. Reprod. Immunol. 61 (2009) 346-359.

[6] A.B. Karabulut, A. Kafkasli, F. Burak, E.M. Gozukara, Maternal and fetal plasma adenosine deaminase, xanthine oxidase and malondialdehyde levels in preeclampsia, Cell. Biochem. Funct. 23 (2005) 279-283.

[7] M.T. Peraçoli, C.F. Bannwart, R. Cristofalo, V.T. Borges, R.A. Costa, S.S. Witkin, et al., Increased reactive oxygen species and tumor necrosis factor-alpha production by monocytes are associated with elevated levels of uric acid in pre-eclamptic women, Am. J. Reprod. Immunol. 66 (2011) 460-467.

[8] V.S. Giorgi, M.T. Peraçoli, J.C. Peraçoli, S.S. Witkin, C.F. Bannwart-Castro, Silibinin modulates the $\mathrm{NF}-\kappa \mathrm{B}$ pathway and pro-inflammatory cytokine production by mononuclear cells from preeclamptic women, J. Reprod. Immunol. 95 (2012) 67-72.

[9] J.C. Peraçoli, C.F. Bannwart-Castro, M. Romao, I.C. Weel, V.R. Ribeiro, V.T Borges, et al., High levels of heat shock protein 70 are associated with proinflammatory cytokines and may differentiate early- from lateonset preeclampsia, J. Reprod. Immunol. 100 (2013) 129-134.

[10] I. Udenze, C. Amadi, N. Awolola, C.C. Makwe, The role of cytokines as inflammatory mediators in preeclampsia, Pan. Afr. Med. J. 20 (2015) 219.

[11] G. Hasko, B.N. Cronstein, Adenosine: an endogenous regulator of innate immunity, Trends Immunol. 25 (2004) 33-39. 
[12] C.I. Ezeamuzie, I. Khan, The role of adenosine $A_{2}$ receptors in the regulation of TNF- $\alpha$ production and $\mathrm{PGE}_{2}$ release in mouse peritoneal macrophages, Int. Immunopathol. 7 (2007) 483-490.

[13] A. Adams, R.A. Harkness, Adenosine deaminase activity in thymus and other human tissues, Clin. Exp. Immunol. 26 (1976) 647-649.

[14] O. Levy, M. Coughlin, B.N. Cronstein, R.M. Roy, A. Desai, M.R. Wessels, The adenosine system selectively inhibits TLR-mediated TNF- $\alpha$ production in the human newborn, J. Immunol. 177 (2006) 1956-1966.

[15] O.O. Oladipo, B.B. Afolabi, A.O. Okorodudu, Adenosine deaminase activity in subjects with normal pregnancy, pregnancy-induced hypertension and preeclampsia, West Afr. J. Med. 28 (2009) 161-164.

[16] Y. Yoneyama, R. Sawa, S. Suzuki, D. Doi, K. Yoneyama, Y. Otsubo, et al. Relationship between plasma malondialdehyde levels and adenosine deaminase activities in preeclampsia, Clin. Chim. Acta 322 (2002) 169-173.

[17] Y. Yoneyama, R. Sawa, S. Suzuki, A. Miura, H. Kobayashi, D. Doi, et al., Relation between adenosine deaminase activities and cytokine-producing $\mathrm{T}$ cells in women with preeclampsia, Clin. Biochem. 35 (2002) 303-306.

[18] Y. Yoneyama, R. Sawa, S. Suzuki, Y. Otsubo, A. Miura, Y. Kuwabara, et al., Serum adenosine deaminase activity in women with pre-eclampsia, Gynecol. Obstet. Invest. 54 (2002) 164-167.

[19] A. Kafkasli, A.B. Karabulut, R. Atmaca, R. Laurini, Clinical correlation between adenosine deaminase activity and pre-eclampsia severity, J. Int. Med. Res. 34 (2006) 247-255.

[20] Z. Kurdoglu, H. Ozkol, M. Kurdoglu, M. Kamaci, Evaluation of the relationship between adenosine deaminase, myeloperoxidase, cholinesterase preeclampsia severity, and neonatal outcomes, Clin. Exp. Hypertens. 34 (2012) 493-497.

[21] J.C. Peraçoli, M.V. Rudge, M.T. Peraçoli, Tumor necrosis factor-alpha in gestation and puerperium of women with gestational hypertension and preeclampsia, Am. J. Reprod. Immunol. 57 (2007) 177-185.
[22] S.A. Bainbridge, J.M. Roberts, Uric acid as a pathogenic factor in preeclampsia, Placenta 29 (Suppl A) (2008) S67-S72.

[23] A.C. Martin, M.A. Brown, Could uric acid have a pathogenic role in preeclampsia?, Nat Rev. Nephrol. 6 (2010) 744-748.

[24] C. Escudero, P. Bertoglia, F. Muñoz, J.M. Roberts, Rev. Med. Chil. 141 (2013) 895-902.

[25] O.H. Lowry, N.J. Rosebrough, A.L. Farr, R.J. Randall, Protein measurement with the Folin-Phenol reagent, J. Biol. Chem. 193 (1951) 265-275.

[26] S.A. Bainbridge, F. von Versen-Höynck, J.M. Roberts, Uric acid inhibits placental system A amino acid uptake, Placenta 30 (2009) 195-200.

[27] C. Jin, R.A. Flavell, Molecular mechanism of NLRP3 inflammasome activation, J. Clin. Immunol. 30 (2010) 628-631.

[28] F. Martinon, V. Petrilli, A. Mayor, A. Tardivel, J. Tschopp, Gout-associated uric acid crystals activate the NALP3 inflammasome, Nature 440 (2006) 237-241.

[29] S.R. Kingsbury, P.G. Conaghan, M.F. McDermott, The role of the NLRP3 inflammasome in gout, J. Inflamm. Res. 4 (2011) 39-49.

[30] M.L. Matias, M. Romão, I.C. Weel, V.R. Ribeiro, P.R. Nunes, V.T. Borges, et al., Endogenous and uric acid-induced activation of NLRP3 inflammasome in pregnant women with preeclampsia, PLoS ONE 10 (2015) e0129095.

[31] B. Levine, N. Mizushima, H.W. Virgin, Autophagy in immunity and inflammation, Nature 469 (2011) 323-335.

[32] T.T. Kanninen, A. Jayaram, S. Jaffe Lifshitz, S.S. Witkin, Altered autophagy induction by sera from pregnant women with preeclampsia: a case-control study, BJOG 121 (2014) 958-964.

[33] P. Kuballa, W.M. Nolte, A.B. Castoreno, R.J. Xavier, Autophagy and the immune system, Annu. Rev. Immunol. 30 (2012) 611-646.

[34] C.P. Wheeler, D.L. Yudilevich, Transport and metabolism of adenosine in the perfused guinea-pig placenta, J. Physiol. 405 (1988) 511-526.

[35] U. Perni, D. Sezen, A.M. Bongiovanni, I.M. Linhares, D. Skupski, S.S. Witkin, Endogenous adenosine down-modulates mid-trimester intraamniotic tumor necrosis factor- $\alpha$ production, Am. J. Reprod. Immunol. 62 (2009) 232-237. 